\title{
Expressão epidemiológica de outras doenças sexualmente transmissíveis entre portadores de AIDS*
}

\author{
Epidemiological expression of other sexually transmitted diseases among AIDS \\ patients
}

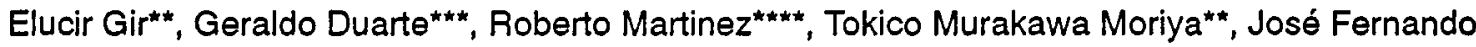

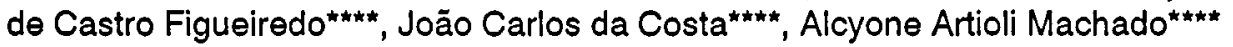

\begin{abstract}
GIR, E. et al. Expressão epidemiológica de outras doenças sexualmente transmissíveis entre portadores de AIDS. Rev. Saúde Pública, 28:93-9, 1994. Objetivou-se avaliar a fireqüência de outras doenças sexualmente transmissiveis (DST) em pacientes portadores de AIDS, identificando-se suas associações epidemiológicas e possíveis relações com as categorias de exposição ao vírus. Os dados foram coletados dos prontuários médicos, identificando-se as DST com base em dados de anamnese, exame físico e exames laboratoriais. Dos portadorcs de HIV/AIDS, atendidos no hospital estudado, de janeiro de 1986 a janeiro de 1992, 207 constituíram a amostra estudada. Dos pacientes estudados, 88 (42,5\%) apresentaram alguma DST e $119(57,5 \%)$ não, resultando proporção de pacientes com DST/pacientes sem DST igual a 0,7. As DST mais prevalentes foram hepatite $\mathrm{B}(33,3 \%)$, sifilis $(30,3 \%)$ e gonorréia $(12,9 \%)$. Quanto às categorias de exposição dos indivíduos ao HIV, a mais prevalente foi a sangüínea $(44,9 \%)$, seguida pela sexual $(21,3 \%)$, sexual e sangüinea $(17,9 \%$ ) e indeterminada em $15,9 \%$. Comparando particularmente as categorias de transmissão sexual e sangüinea do HIV e a presença de outras DST, estas foram significativamente mais freqüentes nos casos cuja categoria de exposição referida foi a sexual.
\end{abstract}

Descritores: Doenças sexualmente transmissíveis, epidemiologia. Síndrome de inunodeficiência adquirida, complicações. Fatores de risco.

\section{Introdução}

O século XX assistiu a grandes progressos da humanidade em sua luta contra as doenças transmissiveis, podendo alirmar-se que foi, $\mathrm{cm}$ grande parte, graças aos mesmos que a população mundial passou de 1, 6 bilhão, em 1900, para aproximadamente 6 bilhões, em 1990 (Montagnier $^{18}$, 1991). Antimicrobianos, vacinas, novos métodos de higienização permitiram

- Apresentado no IV Congresso Brasileiro de Doenças Sexualmente Transmissíveis e $1^{2}$ Simpósio Internacio nal de Viceras Genitais e VIFI. Salvador-BA, 1992.

* Departamento de Enfermagem Geral e Especializada da Escola de Enfermagem de Ribeirão Preto da Universidade de São Paulo - Ribeirão Preto, SP - Brasil

**. Departamento de Ginecologia e Obstetricia da Faculdade de Medicina de Ribeirĩo Preto da Universidade de São Paulo - Ribeirño Preto, SP - Brasil

*** Departamento de Clínica Médica da Faculdade de Medicina de Ribeirão Preto - Ribeirão Preto, SP - Brasil Separatas/Reprints: E. Gir - Av. Bandeirantes, $3900 \cdot 14040-$ 902 - Ribeirão Preto, SP - Brasil

Edição subvencionada pela FAPESP. Processo 94/0500-0 a redução de muitas dessas enfermidades, como por exemplo, a raiva, a febre tifóide, a poliomielite, o tétano e a variola. Entretanto, as doenças sexualmente transmissiveis (DST) recrudesceram a partir dos anos 60, assumindo características de sério problema de saúde pública.

Segundo Belda ${ }^{2}$ (1985), até a década de 50, pequeno número de doenças infecciosas era reconhecido como de transmissão sexual, salientando a sífilis, a gonorréia, o cancro mole, a donovanose e o linfogranuloma venéreo. A partir dos anos 60 novos patógenos foram também responsabilizados e implicados como de transmissão sexual, entre eles a Chlamydia trachomatis, Mycoplasma hominis, Ureaplasma urealyticum, Gardnerella vaginalis e virus da hepatite B (HBV).

Na década de 80 , o vírus da imunodeficiência humana (IIIV) veio integrar a lista das DST, agravando os problemas já existentes na medida em que a forma mais comum de transmissão, na maioria dos países, é a relação sexual com penetração e ejaculação em coito não protegido (Glasel $\left.^{10}, 1991\right)$. 
Devido às particularidades na forma de transmissão e seu aspecto incurável e letal, a AIDS tem assumido caráter prioricário, relegando as demais DST a planos secundários (Cruz Rios ${ }^{7}, 1991$ ).

Evidências clínicas e razões teóricas sugerem que algumas DST podem facilitar a transmissão sexual da infecção pelo HIV, atuando como fator de risco, devido ao aumento da capacidade do portador de HIV infectar outras pessoas. A maioria das DST provoca inflamação e/ou ulccração genital da pele ou mucosas, facilitando assim a disseminação do HIIV (Ahrtag ${ }^{1}$, 1989).

Associadas ao reconhecimento da possibilidade de transmissão sexual de um grande número de microrganismos, ressaltam-se profundas modificações no comportamento sexual das pessoas, o que contribuiu sobretudo para alterar de maneira significante o quadro dessas doenças, tanto nos seus aspectos epidemiológicos quanto nos clínicos-laboratoriais (Duarte8, 1989).

O objetivo do presente estudo foi avaliar a freqüência de outras DST em pacientes infectados pelo HIV, atendidos em hospital localizado em Ribeirão Preto, SP, identificando suas associações epidemiológicas e possíveis relações com as categorias de exposição ao vírus.

\section{Casuística e Metodologia}

De janeiro de 1986 a janeiro de 1992 foram atendidos 916 indivíduos infectados pelo HIV no hospital estudado. Destes, $630(68,8 \%)$ foram identificados como tendo AIDS, grupo IV, e 286 (31, 2\%) nos grupos I, II ou III, segundo classificação do Centers for Disease Control ${ }^{4}, 1986$.

Dos pacientes com AIDS (grupo IV), 392 $(62,2 \%)$ foram a óbito nos anos de 1990 a 1991 , dos quais $207(52,8 \%)$ constituíram a amostra do presente trabalho. Considerou-se como critérios de inclusão: ter idade igual ou superior a 15 anos e ter falecido nos anos de 1990 ou 1991.

A investigação foi do tipo retrospectiva e a coleta de dados realizada a partir dos prontuários médicos. Foram identificados os aspectos referentes a: 1. Identificação Pessoal: sexo, idade, procedência, estado civil; 2. AIDS: fator de risco, categoria de exposição (sexual, sangüínea, sexual/sangüínea, indeterminada), setor do hospital em que foi prestado o primeiro atendimento e tempo de evolução até o óbito; 3. Aspectos relacionados às outras DST: sífilis, gonorrćia, linfogranuloma venéreo, condiloma acuminado, herpes genital, hepatite B, cancro mole e uretrite não gonocócica (UNG), buscando registros que informassem se os pacientes já haviam manifestado algumas dessas doenças anteriormente, independente de ser antes ou após a infecção pelo HIV. Como o atendimento clínico foi prestado por uma mesma equipe considerou-se os dados de prontuário de uniformidade satisfatória.

Foram considerados como antecedentes positivos quando havia alguma referência do paciente sobre o diagnóstico prévio de DST, dados do exame físico e de resultados de exames laboratoriais positivos. Esses aspectos foram avaliados isoladamente ou em associação.

Os dados foram analisados estatisticamente utilizando-se freqüências absolutas e relativas e o teste de significância $X^{2}$ para averigüar-se associação entre algumas variáveis.

\section{Resultados e Discussão}

Dos 207 pacientes avaliados, $172(83,1 \%)$ eram do sexo masculino e 35 (16, 9\%) do sexo feminino, correspondendo a uma proporção 5:1. A idade em que ocorreu o óbito variou de 15 a 61 anos. Verificou-se que 101 pacientes (48, 8\%) pertenciam à faixa de 20 a 29 anos e $67(32,4 \%)$ à faixa de 30 a 40 anos.

No Brasil, dos 34.881 casos de AIDS notificados ao Ministério da Saúde ${ }^{17}$ (1993), correspondentes ao período de 1980 a fevereiro de 1993, a razão entre os sexos masculino e feminino é de 6:1 e a faixa etária predominante de 25 a 34 anos (42,1\%), sendo as subseqüentes de 35 a 39 anos $(15,8 \%)$ e de 20 a 24 anos $(12,0 \%)$. Tais dados indicam que as características dos pacientes do hospital estudado são, em geral, correspondentes aos dados do Ministério da Saúde, ressaltando-se entretanto que constam como notificados nesse órgão apenas 446 casos em Ribeirão Preto, até fevereiro de 1993, o que vem denotar o sub-dimensionamento dos dados do citado Ministério. Mesmo com este sub-dimensionamento, Ribeirão Preto apresenta o quinto maior coeficiente de incidência por 100.000 habitantes entre as cidades brasileiras.

Quanto ao estado civil, 75,8\% dos indivíduos eram solteiros, e em proporções decres- 
centes tem-se a condição casado, mencionada em $16,4 \%$, e as condições viuve'z, desquitado, divorciado e amasiado totalizando 7,7\% dos casos. Esses dados são difíceis de serem avaliados, pois implicam profunda análise sociológica. $O$ baixo percentual de pessoas casadas, nesta casuística, pode induzir ao raciocínio equivocado de que pessoas com esse tipo de vínculo tenham menos $\Lambda$ IDS. Uma avaliação mais aprofundada e específica se faz necessária para dirimir essas dúvidas, uma vez que no cotidiano profissional vivencia-se muitas siluações revcladoras de infidclidade conjugal, configurando situações de clevado risco para a infecção pelo IIIV.

No que se refere à procedencia, obscrvouse que 146 pacicntes $(70,5 \%)$ cram de Ribcirão Preto, $56(27,1 \%)$ de outras cidades do Istado de São Paulo e $5(2,4 \%)$ oriundos de outros Estados brasilciros. Esses números refletcm o papel terciário do hospital estudado, atendendo pessoas do município, de outras regiões e até de outros estados. Esses cncaminhamentos mui- tas vezes se fazem por absoluta falta de suporte hospitalar cm outras regiốcs c por ser Ribcirão Preto um ecntro relativamente diferenciado no atendimento específico desses pacientes, justificando o clevado númcro de pacientes $\mathrm{cm}$ estado precário de saúde nesta casuística.

Quanto aos fatores de risco (Tabela 1), o mais prevalente foi a utilização de droga cndovenosa (EV) alcançado 39,6\%, seguido por droga EV + promiscuidade sexual $(10,7 \%)$, homossexualidade + promiscuidade sexual $(8,7 \%)$ e uranslusão sangúnca $(3,9 \%)$. Em um númcro de casos relativamente elevaclo (33 ou 15,9\%) o fator de risco não pode ser determinado, por insuficiéncia de clados nos prontuários médicos ou em decorrência do mau estado geral do paciente no momento do alendimento.

$\Lambda$ s caractcrísticas epidemiológicas da presente casuística apontam no sentido de que a transmissão sangǘnca, decorrente da utilização dc clroga EV, ć principal forma de transmissão $\mathrm{cm}$ nosso mcio como fitor isolado $(39,6 \%)$,

Tabela 1 - Distribuição de 207 casos de AIDS atendidos em hospital de Ribeirão Preto, SP, segundo fator de risco.

\begin{tabular}{|c|c|c|}
\hline \multirow{2}{*}{ Fator de Risco } & \multicolumn{2}{|c|}{ Total } \\
\hline & № & $F(\%)$ \\
\hline Usuário de droga EV & 82 & $(38,6)$ \\
\hline Usuário de droga EV + promiscuidade sexual & 22 & $(10,7)$ \\
\hline Homessexual + promiscuidade sexual & 18 & $(8,7)$ \\
\hline Transfusào sangüinea & 8 & $(3,9)$ \\
\hline Promiscuidade sexual & 6 & $(2,9)$ \\
\hline Bissexual + usuários de droga EV + promiscuidade sexual & 5 & $(2,4)$ \\
\hline Homossexual + parceiro anti-HIV positivo & 4 & $(1,9)$ \\
\hline Promiscuidade sexual + relação com prostitutas & 4 & $(1,9)$ \\
\hline Usuários de droga EV + presidiário & 4 & $(1,9)$ \\
\hline Parceiro(a) anti-HIV positivo & 3 & $(1,4)$ \\
\hline Bissexual + promiscuidade sexual & 3 & $(1,4)$ \\
\hline Presidiário & 2 & $(1,0)$ \\
\hline Usuário de droga EV + parceiro(a) anti-HIV positivo & 2 & $(1,0)$ \\
\hline Homossexual + usuário de droga EV & 2 & $(1,0)$ \\
\hline Relaçāo com prostitutas & 1 & $(0,5)$ \\
\hline Usuário de droga EV + transfusão sangüinea & 1 & $(0,5)$ \\
\hline Parceiro sexual usuário de droga $\mathrm{EV}$ & 1 & $(0,5)$ \\
\hline Homossexual + promiscuidade sexual + usuário de droga EV & 1 & $(0,5)$ \\
\hline Relação sexual com prostitutas + parceiros(a) anti-HIV & 1 & $(0,5)$ \\
\hline Homossexual + transfusäo sangüinea & 1 & $(0,5)$ \\
\hline Promiscuidade sexual + transfusāo sangüinea + parceiro(a) anti-HIV positivo & 1 & $(0,5)$ \\
\hline Homossexual + promiscuidade sexual + parceiro(a) anti-HIV positivo & 1 & $(0,5)$ \\
\hline Parceiro sexual promiscuo & 1 & $(0,5)$ \\
\hline Promiscuidade sexual + transfusão sangüinea & 1 & $(0,5)$ \\
\hline Não consta no prontuário & 17 & $(8,2)$ \\
\hline Indeterminado & 16 & $(7,7)$ \\
\hline Total & 207 & $(100)$ \\
\hline
\end{tabular}

EV - Endovenosa 


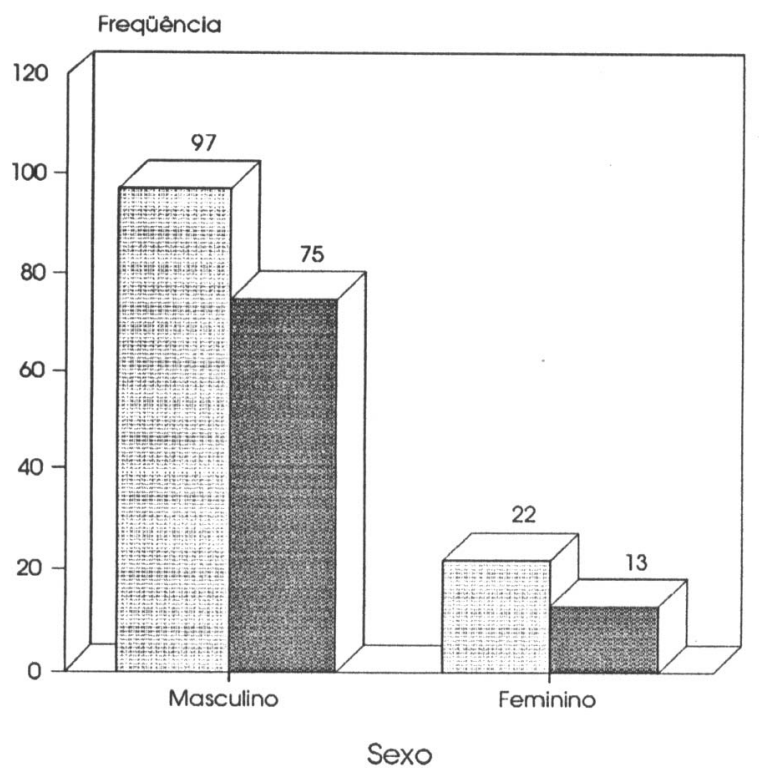

$$
\begin{array}{lll}
x^{2} \text { crítico }=3,84 \quad & x^{2} \text { calculado }=0,48 \quad \alpha=0,05
\end{array}
$$

Figura 1 - Distribuição de 207 casos de AIDS, atendidos em hospital de Ribeiräo Preto, SP, segundo o sexo e a ocorrência de outras DST

salientando-se que em 38 casos $(18,4 \%)$ o uso de droga EV esteve associado a outros fatores de risco. Esses dados concordam com os resultados referidos por Duarte e col. ${ }^{9}$ (1991).

Em relação ao sexo e a ocorrência de outras DST (Fig. 1), observou-se que $43,6 \%$ dos homens tinham antecedentes de DST. Entre as mulheres observou-se que $37,1 \%$ também apresentaram tais doenças. Comparando-se a freqüência dessas doenças entre os indivíduos dos sexos masculino e feminino, não se verificou diferença estatisticamente significante, corro- borando (1990-1991) os dados divulgados por Costa e col. ${ }^{6}(1991)$.

A proporção encontrada entre o número de pacientes que apresentaram alguma DST (88) e o número de indivíduos que não apresentaram tal diagnóstico (119) foi de 0,7 , razão seguramente expressiva.

Dentre as DST que mais se associaram a pessoas infectadas pelo HIV, a mais prevalente foi hepatite B $(33,3 \%)$, seguida por siffilis $(30,3 \%)$, gonorréia $(12,9 \%)$, herpes genital $(9,8 \%)$, condiloma acuminado $(5,3 \%)$, cancro mole $(4,5 \%)$, UNG $(3,0 \%)$ e linfogranuloma venéreo $(0,8 \%)$, totalizanclo 132 diagnósticos de DST cm 88 pacientes (Tabcla-2). Relletindo sobre esse total de diagnósticos $\mathrm{cm}$ relação ao total geral de pacientes estudados, observou-se uma proporção de 0,6 DST para cada indivíduo avaliado. A identificação dessas doenças se baseou em dados de anamnese, do exame físico e de resultados laboratoriais. Em relação ao diagnóstico através de exames laboratoriais, considerou-se como hepatite a presença no soro de ao menos um antígeno ou anticorpo relacionado ao HBV. Portanto, o total de 44 pessoas identificadas como indivíduos que tiveram antecedentes de hepatite $B$, não necessariamente indicam que todos tiveram a doença clinicamente manifesta, podendo expressar apenas infecção subclínica.

Esses dados mostram claramente a importância das DS'T serem consideradas como possíveis fatores de risco, predispondo à infecção pelo IIIV, ou mesmo como marcadores/indicadores clínicos que podem identificar pessoas perlencentes a grupos/comportamentos de riscos, concordando portanto com diversos estu-

Tabela 2 - Distribuição de 132 episódios ou casos de doenças sexualmente transmissiveis, diagnosticadas em 88 pacientes portadores de AIDS atendidos no hospital de Ribeirão Preto, SP (1990-1991).

\begin{tabular}{lccccc}
\hline & $\begin{array}{c}\text { Exame Laboratorial } \\
\text { Positivo }\end{array}$ & Exame Fisico & $\begin{array}{c}\text { Referencial } \\
\text { Paciente }\end{array}$ & $\begin{array}{c}\text { Exame Laboratorial+ } \\
\text { Exame Fisico }\end{array}$ & Total \\
\hline Hepatite B & 41 & - & 3 & - & $44(33,3 \%)$ \\
Sífilis & 15 & 1 & 15 & 9 & $40(30,3 \%)$ \\
Uretrite não gonocócica & - & 1 & 16 & - & $17(12,9 \%)$ \\
Herpes genital & - & 12 & 1 & - & $13(9,8 \%)$ \\
Condiloma acuminado & - & 6 & 1 & - & $7(5,3 \%)$ \\
Cancro mole & - & 3 & 4 & 1 & $6(4,5 \%)$ \\
Uretrite näo gonocócica & - & - & 1 & - & $4(3,1 \%)$ \\
Linfogranuloma venéreo & - & - & & - & $1(0,7 \%)$ \\
Total & & & & $132(100 \%)$ \\
\hline
\end{tabular}


dos realizados em países ocidentais e africanos (Cameron e col. ${ }^{3}$ 1987; Handsfield e col. ${ }^{12}$, 1987; Moss e col. ${ }^{\text {i9 }}$, 1987; Greenblatt e col. ${ }^{11}$ ', 1988; Simonsen e col. ${ }^{22}$, 1988; Pepin e col. ${ }^{21}$ ', 1989; Chiasson e col. ${ }^{5}$, 1990; Harkess e col. ${ }^{13}$, 1990; Kwan e Lowe $\left.{ }^{14}, 1992\right)$.

Foram identificados como pessoas que nunca apresentaram nenhuma $D^{\prime} \Gamma, 119$ pacientes. Entretanto, dentre esses casos alguns não puderam ter identificado o seu passado venéreo em decorrência do precário estado geral no momento do atendimento. Além disso, verificou-se que $51,7 \%$ faleceram com menos de 2 meses de evolução, salientando-se que destes, $39,1 \%$ apresentaram um tempo de vida inferior a 30 dias (Fig. 2).

Associando-se as informações de que a maioria apresentou período curto de seguimento na instituição e o estado geral do paciente era grave, requerendo atendimento de urgência, entende-se que provavelmente a investigação sobre outras DST, nestas circunstâncias, tornase não prioritária e até mesmo não faclível.

No que se refere ao hospital em estudo, ressalta-se que este apresenta duas frentes de atendimentos situadas em locais distintos: a Unidade de Emergência (UE), localizada no centro da cidade de Ribeirão Preto, e o hospital

tempo (mês)

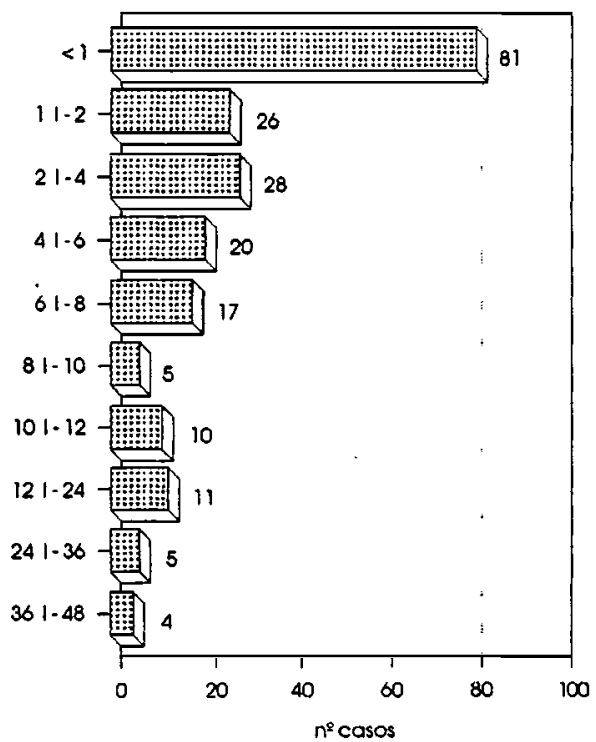

Figura 2 - Distribuição de 207 casos de AIDS atendidos no hospital de Ribeirão Preto, SP (1990-1991), segundo o tempo de seguimento médico nesta instituição. localizado no campus universitário onde é prestado atendimento ambulatorial e internações de rotina. Em decorrência desta particularidade, $60,4 \%$ desses pacientes foram atendidos inicialmente na UE, mesmo por que o estado clínico de grande parte desses indivíduos demandava atendimento de urgência, por apresentarem graves quadros respiratórios, neurológicos, intestinais ou dermatológicos.

Por ocasião do primeiro atendimento, um total de $35,7 \%$ dos pacientes já sabiam que eram contaminados pelo HIV e os demais tiveram seu diagnóstico realizado no hospital.

No que diz respeito às categorias de exposição dos individuos ao IIIV, verificou-se que em 93 pacientes $(44,9 \%)$ a categoria de exposição foi sangüínea, seguida da transmissão sexual $\mathrm{cm} 44(21,3 \%)$, sexual e sangüínea em 37 $(17,9 \%)$ e indeterminado em 33 pacientes $(15,9 \%)$. Quando comparados os dados acumulados do Ministério da Saúde ${ }^{17}$ (1993) em relação à categoria de exposição, verifica-se que os dados gerais do Brasil indicam a categoria sexual como a mais prevalente $(61,3 \%)$ Parker e Carballo $^{20}$, 1991). Segundo Mhloyi ${ }^{15}$ (1992) a relação sexual pode não ser o modo mais eficiente de transmissão, mas constitui-se na mais comum e a mais amenizável forma de transmissão, visto que a transmissão heterossexual está aumentando em todos os países do mundo, em proporções alarmantes.

Ao se analisar as categorias de exposição do IIIV em relação à presença ou não de outras DST, verificou-se que em relação à transmissão sexual isoladamente, $65,9 \%$ dos indivíduos apresentavam pelo menos uma DST, ao passo que, nessa categoria de exposição, apenas $34,1 \%$ não apresentaram essas doenças. Por outro lado, ao se estudar a transmissão sangüínea isoladamente, observaram-se dados opostos, ou seja, 33,3\% apresentaram outras DST e em $66,7 \%$ não foi diagnosticada esta associação. Estas diferenças analisadas através de teste não paramétrico (qui-quadrado) mostraram significância estatística (Tabela 3), evidenciando uma correlação mais estreita entre categoria de exposição sexual e AIDS quando comparado com a categoria de exposição sangüínea.

Esses achados, portanto, constituem motivos para os autores evidenciarem que as DST estão associadas aos portadores de HIV/AIDS, 
Tabela 3 - Distribuição de 207 casos de pacientes atendidos no hospital de Ribeirão Preto, SP (1990-1991), segundo as categorias de exposiçäo ao HIV e a ocorrência ou não de outras DST

\begin{tabular}{lccc}
\hline & \multicolumn{2}{c}{ DST } & Total \\
\cline { 2 - 3 } Categoria de & $\operatorname{Sim}$ & Não & \\
Exposição ao HIV & $n^{2} f(\%)$ & $n^{2} f(\%)$ & $n^{2} f(\%)$ \\
\hline "Sexual & $29(65,9)^{*}$ & $15(34,1)^{*}$ & $44(100)$ \\
* Sangüinea & $31(33,3)^{*}$ & $62(66,7)^{*}$ & $93(100)$ \\
Sexual/Sangüinea & $22(59,5)$ & $15(40,5)$ & $37(100)$ \\
Indeterminada & $6(18,2)$ & $27(81,8)$ & $33(100)$ \\
\hline Total & 88 & 119 & $207(100)$ \\
\hline$x^{2}$ crítico $=3,84$ & $x^{2}$ calculado $=12,7$ & $a=0,05$ &
\end{tabular}

embora não se tenham investigado a ocorrência temporal dessas patologias, em relação à infeceção pelo HIV, se antes ou após.

De acordo com o Ministério da Saúde ${ }^{16}$ (1989), existem fortes razões teóricas, assim como evidências clínicas para se afirmar que certas DST são fatores de risco na transmissão do HIIV, ou seja, clas podem facilitar a transmissão do vírus de um pessoa infectada a seu parceiro durante a relação sexual. Lspecula-se, ainda, que a alta incidência de algumas DS $\Gamma$, registrada em vários centros urbanos da África Tropical, pode ser um fato importante associado à rápida propagação do IIIV na região, ocorrida através de relações hetcrossexuais.

Como referem Werne c Grusin ${ }^{23}$ (1981), a hipervalorização da AIDS, as preocupações maiores com herpes e clamídia, desviaram para um plano secundário as clássicas doenças venéreas. Este também é o pensamento de Cruz Rios $^{7}$ (1991), que sugere a nccessidade imperiosa de combate às DST, pois, ontem como hoje, elas se firmam como causa decisiva na clisseminação da AIDS, ficando portanto o controle da AIDS também na dependência das DST.

Desta forma, as tentativas de se controlar a disseminação do HIV, sem a implementação de medidas paralclas que visem à prevenção e ao controle das outras DST, têm menos possibilidades de screm eficazes.

No momento, a atençĩo governamental $\mathrm{cm}$ todos os países tem se voltado prioritariamente para AIDS. Entretanto, consideram-se pertinentes as palavias de Woscofl ${ }^{24}$ (1989) de que a AIDS é uma enfermidade de transmissão scxual, mas não é a AIDS que favorece as DS'T c sim as DST que favorecem a AIDS.
Logo, não se justifica claborar projetos especílicos sobre $\Lambda$ IDS, devendo-se também dirigir e somar esforços à luta contra as DS'T clássicas que, aincla hoje, assumcm importância em nível de saúde pública mundial.

A proporção de 0,6 DS'I' para cada paciente com AIDS, encontrado na presente casuística, veio reforçar a inquictação de que a pesquisa e a associação das outras DS'l' e a AIDS não devem ser subestimaclas. Tampouco, as pessoas devem continuar hipervalorizando projetos e ações isolaclas sobre AIDS uma vez que há razões tcóricas e clínicas para se acreditar que a prevenção e o controle da $\Lambda$ IDS estão umbilicalmentc relacionados com a prevenção e o controle clas outras DST e vice-versa.

GIR, E. et al. [Epidemiological expression of other sexually transmitted discases among AIDS patients. Rev. Saúle Páblica, 28: 93-9, 1994. This study was carried out in order to cstimate the frequence of other sexually transmitted discases (STD) among AIDS patients and to identify their epidemiological association and possible relations to the groups most exposed to the virus. The data were collected from the medical case histories and the SID were identified on the basis of data provided by anamuesis, pliysical examination and laboratory examinations. Of the total of IIIV/ADDS patients assisted at the hospital studied (S.Paulo State, Brazil), between January 1986 and January 1992, 207 were included as sample subjects for this survey. Of the patients studied, $88(12.5 \%)$ had some other SID and $119(57.5 \%)$ had no other SID, equivalent to a proportion of 0.7 STD patients-S'D to each non-STD patient. The most prevalent SID identificd were hepatitis $B(33.3 \%)$, syphilis (30.3\%) and gonorthoea (12.9\%). Concerning the means of transmission by which the patients had probably been infected with IIIV, blood transmission was the most prevalent (14.9\%); followed by sexual contact (21.3\%); sexual/blood (17.9\%); in $25.9 \%$ it was 
undetermined. In a particular comparison of sexual and blood transmisson and the presence of olher S'TD a statatiscal dilference in those cases that were exposed to sexual transmission was observed.

Keywords: Sexually transmitted diseases, epiclemiology. Acquired imumodeficiency syndrome, complications. Risk factors.

\section{Referências Bibliográficas}

1. AIIRTAG. O que você sabe sobre doençăs sexualmente transmissívcis? Ação Anti-AIDS, (6) 1989.

2. BEI.DA, W. Doenças sexualmente transmissiveis. Inform. Uiolog. Enctite, (16):1-2, 1985.

3. CAMERON, W.D.; PLUMMLR, I.A.; SIMONSEN, J.N.; NDINYA - ACIIOLA, J.O.; D'COS'l'A, L.J.; PIO'I', P. et al. Female to male heterosexual transmision of IIIV infection in Nairobi. In: Intcrnational Conference on AIDS, 3rd, Washington DC, 1987. Abstract volume. Washington DC, 1987. p.25.

4. CENTERS IOOR DISLASE CONIROI.. Classification system for Human 'I' - Lymphorropic Virus type Ill/Lympladenopathy: associated virus infections. Ann. Intern. Med., 105: 231-7, 1986.

5. CIIIASSON, M.A.; STONIBUURNER, R.L.; LIISSON, A.R.; IHLDEBBANT, D.S.; EWING, W.E.; SCIIUITZ, S.; JAFFE, H.W. Risk factors for human immunodeficiency virus type (IIV - 1) infection in paticnts at a sexually transmitted discase clinic in Now Yotk City. Am. J. Fpidomiol., 131: 208-20, 1990.

6. COSTA, J.C.; DUARTE, G.; MACILADO, A.A.; FIGULIREDO, J.I.G.; MARTINEZ, R.; GIR, E.; MORIYA, T.M. Prevalencia de otras "LETS" cn pacientes con infección por el "VIII" en Ribciño l’reto (Brasil). In: Congresso Latino Americano de Enfermedades de Transmisión Sexual, $7^{2}$, Santo Domingo, 1991. Programa.p.28-17.

7. CRUZ RIOS, I. Editorial. Bol Iıjorm. UNIÓN, 16(62):13, 1991 .

8. DUAR'TE, G. Docnças sexualmente transmissíveis e gravidez. In: Viggiano, M.G.C. Conclutas em obstetircia, $3^{2}$ ed. Rio de Janciro Athencu, 1989. p. 411-46.

9. DUAR'TE, G.; MUSSIPINIATA, M.M.; DEL, LAMA, J.; TAKEDA, PASTI, M.J.; COSIA, J.C. Valor do questionário específico nas identificações de parturientes de risco para a infecçĩo pelo virus da imunodeficiência humana (IIIV). J. Bras. Ginecol., 101:169$74,1991$.

10. GLASEL, M. Prúticas sexuais de alto risco na transmissão da AIDS/SIDA. In: De Vita Jr, V.T.; IIellman, S.; Rosemberg, S.A. AIDS/SIDA: eliologia, aliagnóstico, tratamento e prevenção. $2^{2}$ ed. Rio de Janciro, Revinter, 1991. p.363.76.

11. GREENIBLATI, R.M.; LUKEIIART, S.A.; PLUMMIR, F.A.; QUINN, 'T.C.; CRIICIILOW, C.W.; ASIILEY, R.L.;
D'COSIA, L.J.; NDINYA-ACIIOLA, J.O.; COREY, L.; RONALD, A.R. Genital ulceration as a risk factor for IIIV infection. AlDS, 22:4́ 7-50, 1988.

12. IIANSIILID, II.II,; ASIILLY, R.L.; ROMPALO, A.M.; STAMM, W.E.; WOOD, R.W.; COREY, L. Association of anogenital ulcer discase with human immunodeficiency virus infection in homosexual men. In: International Conference on AIDS, 3rd., Washington DC, 1987. Abstract volume. Washington, 1)C, 1987. p.206.

13. II man immunodeficicncy virus infection and targeting prevention. South. Med. J., 83:1253-5, 1990.

11. KWAN, D.J. \& LOWE, I.C. Acquired immunodeficiency syndrome: a vencral discase. Urolog. Clin. North. America, 19:13-21, 1992.

15. MIIOYY, M.M. The psychosocial aspects of AIDS. In: Giraldo, G.; Rossi, G.B.; Bcth-Gitaldo, E.; ChiccoBianchi, L.; Dianzani, l.; Giraldo, G.; Venuni, P. Scicnce chatling AIDS. Bassel, Karger, 1992. p.159-74.

16. MINIS'ÍERIO DA SAÚDl: Doençals sexualmente transmissíveis. Bol. Eptidemiol., 1(2) 1989.

17. MINISIÉRIO DA SAUUDL. Scmana cpiclemiológica. AIDS Bol. Eipictemiol, 6(1) 1993.

18. MONTAGNIER, L. Medicina do futuro vai ser privilégio clos países ricos. Folha de São Paulo, Caderno de Ciência, 27 lov. 1991. p.14.

19. MOSS, A.R.; OSMOND, D.; BACIIEITI, P.; CIIERMANN, J.; BARRÍ:SINOUSSI, F.; CARLOS, N.J. Risk factors for AIDS and IIIV seropositivity in homosexual men. Am. J. Epialemiol., 125:1035-47, 1987.

20. PARKIIR, HI. \& CARIBALI.O, M. Sexual culture, IIIV transmission and IIIV rescarch. J. Sex. Res., 28:77-8, $19) 1$.

21. PIPPIN, J; PLUMMIIR, F.A; IBRUNIIAM, R.C.; PIOT, P.; CAMERON, D.W.; RON MLD, A.R. Ihe interaction of IIIV infection and other sexually transmitted discases: on opportunity for prevention. AIDS, 3:39, 1989 .

22. SIMONSIEN, J.N.; CAMERON, W.D.; GAKINYA, M.N.; NDINYA-ACIIOLA, J.O.; D'COSIA, L.J.; KARASIRA, P.; CIIEANG, M.; MUTH, M.; RONAI.D, A.R.; PIOT, P.; PLUMMER, F.A. I Iuman immunodeficiency virus infection among men with sextally transmilted discases: experience from a Center in Africa. N. Engl. J. Heal, 319:274-8, 1988

23. WLRNE, C.S. \& GRUSIN, I.. Failure to diagnose secondary syphilis. Conn. Afel., 48:769-70, 1984.

24. WOSCOI:I, A. Discurso de abertura do VII Congresso da Unión Latlino Americana Contra Enfermedades de Transmisión Sexual (UIACETS), La ILabana, Cuba, 1989. Bol. Infor: UNIÓN, 14(55):1-2, 1989.

Recebiclo para publicação em 29.4.1993 Reapuesentado em 12.11.1993

Aprovalo para publicação em 26.11.1993 